\title{
Rogowski Coil for Current and On-Temperature Monitoring Overhead Transmission Lines
}

\author{
Ch. Bhaskar \\ PG Scholor \\ Department of ECE \\ Vardhaman College of Engineering \\ Hyderabad, India
}

\author{
K.Harikrishna, Ph.D \\ Professor \\ Department of ECE \\ Vardhaman College of Engineering \\ Hyderabad, India
}

\begin{abstract}
This letter describes the development of a rogowski coil current and on-chip temperature sensor for evaluating the sag of conductors in high voltage transmission line. A rogowski coil has a air core instead of an iron core. The measurement show excellent linearity with practical no saturation problems. In addition, the rogowski rate highly for electrical isolation from the bus bar, and is light weight with low material cost. The uses reliably proven universal serial bus technology to energize the electronic circuitry. The data collected together with sag information will provide support for the development of an algorithm for estimation conductor sag values.
\end{abstract}

\section{Keywords}

Current sensor, power measurement IC, sag, transmission line, universal serial bus cable.

\section{INTRODUCTION}

A transmission line is a specialized cable or other structure designed to carry alternating current of radio frequency. Transmission lines capacity is the maximum amount of energy or current capable of flowing through the line. When the demand for energy increases, technicians do not raise the current above the designed limit due to concerns about infringing safety standards and affecting performance, such as wire temperature and sag. The sag is an important transmission line parameter, since it is directly related to the current. The conductor temperature does not depend only on the current. It is dependent on environment. Such as wind velocity and direction, air temperature, pollution, rain, and snow conditions and on cable construction [1]. So, transmission line maximum sag characteristic is always aiming at the best safety conditions.

This idea behind this project is that the temperature of the conductor depends on current and weather conditions, but the sag only depends on the conductor temperature, regard less of weather conditions. Therefore, a system capable of measuring the temperature of the conductor, together with the current flowing through it would help technicians determine whether the sag is beyond the safety restrictions, regardless of the current or weather conditions. The final objective of this project is to provide support for the development of an algorithm for the estimation of sag values, using collected temperature and current data together with sag information.

The feasibility of collecting energy from a high current conductor inthe form of a magnetic field, in order to run a small local power supply [2], has been demonstrated. This solution works very well for high current transmission lines but there are cases in which the current goes down beyond the capacity of producing any magnetic field strong enough to drive a power supply. In 1997 it has been presented a photonic powered for intelligent sensors, where the sensor was separated from the intelligent control. However, at the time, the photovoltaic cells were very inefficient and, in addition, laser sources were expensive, factors which restricted the system design. A system using a universal serial bus interface to the personal computer. A pc will be display the temperature and current data.

This letter describes a rogowski coil for current and ontemperature sag monitoring of conductors in high voltage transmission line. To make measurements, the system uses reliably proven electronic technology which do not require complex modulation techniques making the overall cost of a single piece of equipment, including deployment and production expenses, reduced. Photonic technology energizes the electronic circuitry and transmits the signals via universal serial bus cable. The data collected, together with the sag information, will provide support for the development of an algorithm for the estimation of conductor sag values.

\section{SYSTEM DESCRIPTION AND TESTS}

The rogowski coil for current and on-temperature monitoring in overhead transmission lines is composed of two subsystems: high voltage is an instrument suite composed of a rogowski current sensor [3], a temperature sensor, a photovoltaic converter, a low -power microcontroller with serial output. In this way, current and temperature are measured, digitized, processed and sent to the receiving system.

Five systemic tests were performed: current calibration; temperature calibration; electromagnetic compatibility (EMI); enclosure protection against water under IP65 protection code and HV supportability under saline rain. For current calibration a current loop, adjusted by a variable transformer, simultaneously excited the prototype sensor and a calibrated current probe. The calibration range was from 100 to 650 Arms, consistent with the transmission line where the system was installed. The temperature was calibrated by an oil bath in the range of $10^{\circ} \mathrm{C}$ to $90^{\circ} \mathrm{C}$ which was also consistent with local temperature. The current and temperature correlation coefficients were 0.9999 and 0.9995 , respectively. The uncertainties were $+0.23 \mathrm{~A}$ at $500 \mathrm{~A}$ and $+0.01^{\circ} \mathrm{C}$ at , both for a $95 \%$ confidence interval. The EMI test was carried out in our laboratory for a magnetic field equivalent to a current of $1000 \mathrm{~A}$, well above the transmission line maximum current and electric field equivalent to a voltage of $25 \mathrm{KV}$, which is well below the nominal voltage of the transmission line, about $40 \mathrm{KV}$ phase-to-ground (equivalent to $69 \mathrm{KV}$ phase-to-phase). The reason for this was the maximum voltage limitation of our high voltage rig. The test for high voltage supportability under saline rain was performed according to [4]. In order to assure the rate of protection established by IP65 enclosures, the test involves subjecting the fully operational prototype to a 
continuous jet of water properly during the test, not showing any faults due to extreme humidity.

A rogowski coil current sensor is a helical coil of wire wrapped around an $\mathrm{AC}$ line conductor and used to measure the flow of electric charge through the conductor. Since a rogowski coil has an air core instead of an iron core, the measurements show excellent linearity with practically no saturation problems. In addition, the rogowski coil rates highly for electrical isolation from the bus bar, and is light weight with low material cost. The rogowski coil is increasingly being implemented when measuring high-current AC power and energy.

The voltage $\mathrm{v}(\mathrm{t})$ that is induced in the rogowski coil is proportional to the rate of change of current $(\mathrm{di}(\mathrm{t}) / \mathrm{dt})$ and is based on Faraday's law. The voltage $v(t)$ produced by a rogowski coil is calculated using Equation 1:

$$
\mathrm{V}(\mathrm{t})=\left(-\frac{A N \mu 0}{l}\right)\left(\frac{d i(t)}{d t}\right)
$$

Where,

$$
\begin{aligned}
& A=\text { Area of each small loop } \\
& N=\text { Number of turns } \\
& l=2 \pi R \text { and is the length of the winding } \\
& \mu_{0}=\text { permeability of free space }
\end{aligned}
$$

When the Ac line current , I, is a50Hz or $60 \mathrm{~Hz}$ sinusoidal, Equation 1 can be simplified to equation 2 .

$$
\mathrm{V}=\mathrm{K} 1
$$

Where
$\mathrm{K}=$ sensitivity constant that represents the output voltage per ampere at $50 \mathrm{~Hz}$ or $60 \mathrm{~Hz}$. A rogowski coil output Voltage/ Ampere at $50 \mathrm{~Hz}$ is $416 \mu \mathrm{V} / \mathrm{A}$.

A rogowski coil interfaced through the power measurement IC CS5490. The current and voltage values are gathering from the rogowski coil The CS5490 is a CMOS power measurement integrated circuit that uses two analog-to-digital converters to measure line voltage and current. The CS5490 calculates active, reactive , and apparent power as well as RMS voltage and current and peak voltage and current. It handles other system-related functions, such as energy pulse generation, voltage sag and swell, over current zero-crossing detection, and line frequency measurement. A separate analog-to-digital converter is used for on-chip temperature measurement. The CS5490 is optimized to interface to current transformers, shunt resistors, or rogowski coils for current measurement, and to resistive dividers or voltage transformers for voltage measurement. Two full-scale ranges are provided on the current input to accommodate different types of current sensors. The CS5490's two differential inputs have a common-mode input range from analog ground(GNDA) to the positive analog supply(VDDA). An on-chip voltage reference (typically 2.4 volts) is generated and provided at analog output, VREF \pm . The digital output (DO) provides a variety of output signals and, depending on the mode selected, provides energy pulses, zero-crossings.

Two types of isolation are provided : a low-speed optical coupler and high-speed digital isolation for UART communication. The high speed digital isolators operate from DC to $150 \mathrm{Mbps}$. The low-speed optical couplers operate to a maximum speed of about $4.8 \mathrm{kHz}$. All the signals supplied to the isolators are available to the microcontroller.

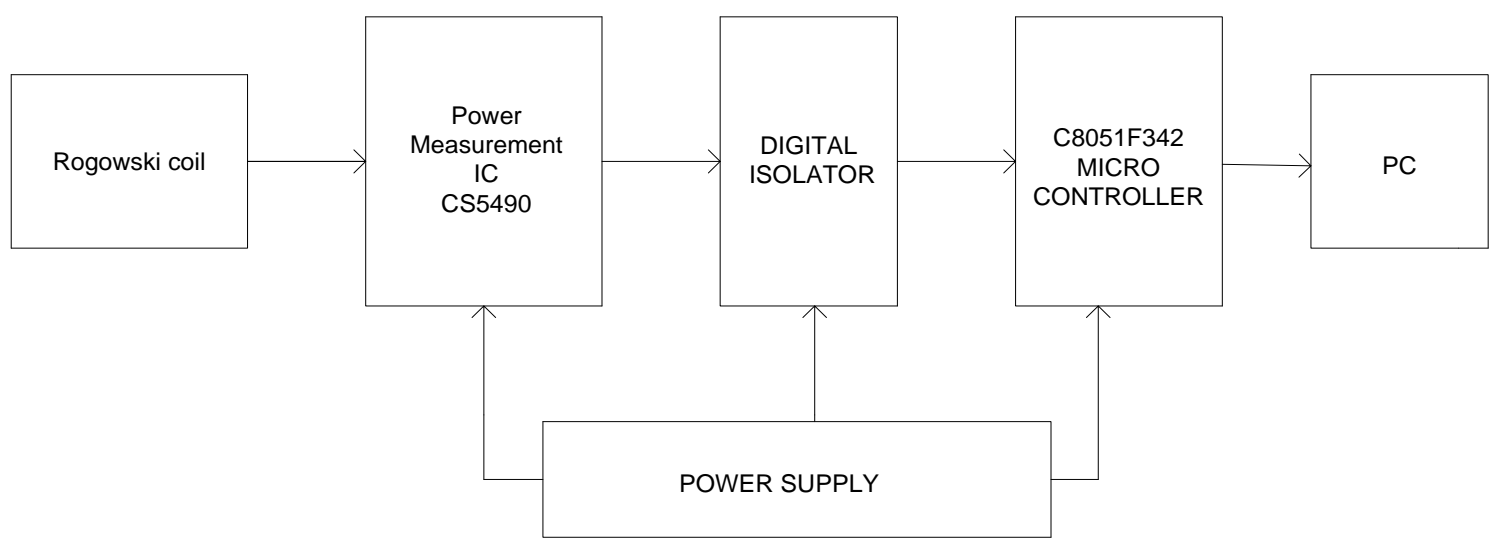

Fig 1: Block diagram of the system. The two subsystems are interconnected by USB cable 

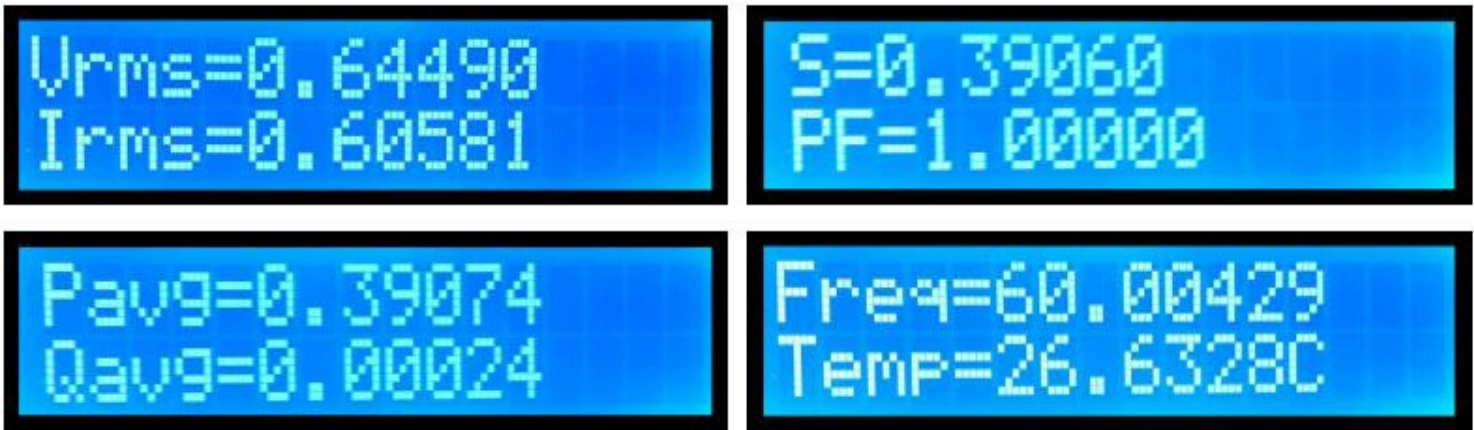

Fig 2: the system output displayed in the PC (or) LCD.

\section{DEPLOYMENT}

Even though digital isolation is provided with analog and digital section the prototype. However, just a few minutes after deployment the prototype presented faults due to the proximity of electric field and data stopped being sent to the base station. The microcontroller situated near the high voltage showed a high susceptibility to EMI.

\section{MALFUNCTIONING MITIGATION}

Digital isolation provided with analog and digital section the prototype is placed in the metallic box. A metallic box with all negative voltages inside the enclosure grounded to the box, therefore shielding the electronic circuitry against the high voltage electric field. Then, the system was taken to an accredited laboratory (CEPEL-Electrical Energy Research center, located in Rio deojaneiro) to test it against EMI, this time up to $138 \mathrm{kV}$. The prototype performed very well without any failure. The system is now operational and waiting for the next installation window in order to monitor current and temperature against sag. It is expected that the data collected, together with the sag information, will provide support for the development of an algorithm for the estimation of conductor sag values.

\section{REFERENCES}

[1] D.A. Douglas and R. Thrash, sag and Tension of conductor - The Electric power Engineering Handbook. Boca raton, FL: CRC Press, 2007, ch. 14.

[2] L.Du, C. Wang, X.Li, L. Yang, Y. Mi, and C.sun, "A novel power supplyof online monitoring systems for power transmission lines",IEEE Trans. Ind. Electron., vol. 57, no. 8, pp.2889-2895, 2010.

[3] Ibrahim A. "Performance improvement of slow wave rogowski coils for high impulse current measurement", IEEE Journal.

[4] "Artificial contamination tests on hv insulators to be used on ac systems", IEC, 1991, publication 507.

[5] "Hybrid optoelectronic sensor for current and temperature monitoring overhead transmission lines" IEEE sensors Journal, Vol.12, No.5 May 2012.

[6] D.A. Ward, J.La T.Exon."using rogowski coil for transient current measurements" 